\title{
Sensitivity of Superficial Cultures in Lower Extremity Wounds
}

Chayan Chakraborti, mD ${ }^{1}$ Christina Le, $\mathrm{ms}^{2}$ Andrew Yanofsky, $\mathrm{MD}^{3}$

\author{
${ }^{1}$ Department of Internal Medicine, Tulane University Health Sciences Center, New Orleans, Louisiana. \\ ${ }^{2}$ Tulane University School of Medicine, New Orleans, Louisiana. \\ ${ }^{3}$ Department of Internal Medicine, George Washington University School of Medicine, Washington, \\ DC.
}

Disclosure: Nothing to report.

BACKGROUND: Superficial wound cultures are routinely used to guide therapy, despite a lack of clear supporting evidence. PURPOSE: To conduct a systematic review of the correlation between superficial wound cultures and the etiology of skin and soft tissue infections.

DATA SOURCES: Medline, EMBASE, CINAHL, Scopus.

STUDY SELECTION: Articles published between January 1960 and August 2009 involving superficial wound cultures and deeper comparison cultures.

DATA EXTRACTION: Two reviewers independently searched for abstracted information pertaining to the microbiology of lower extremity wounds sufficient to calculate the sensitivity and specificity of superficial wound cultures versus comparison cultures.

DATA SYNTHESIS: Data pooled using a random-effects meta-analysis model.

RESULTS: Of 9032 unique citations, 8 studies met all inclusion criteria. Inter-rater reliability was substantial (Kappa $=0.78$ ). Pooled test sensitivity for superficial wound swabs was $49 \%$ (95\% confidence interval [CI], 37-61\%], and specificity was $62 \%$ (95\% CI, 51-74\%). The pooled positive and negative likelihood ratios (LRs) were 1.1 (95\% CI, 0.71-1.5) and 0.67 (95\% CI, 0.520.82). The median number of isolates for surface cultures (2.7, interquartile range [IQR] 1.8-3.2) was not significantly different than that for comparison cultures, (2.2, IQR 1.7-2.9) $(P=0.75)$.

CONCLUSION: Few studies show a strong relationship between superficial wound swabs and deep tissue cultures, and the current data demonstrate poor overall sensitivity and specificity. The positive and negative LRs were found to provide minimal utility in influencing pretest probabilities. Results of this analysis show that wound cultures should not be used in lieu of local antibiograms to guide initial antibiotic therapies. Journal of Hospital Medicine 2010;5:415-420.

() 2010 Society of Hospital Medicine.

KEYWORDS: cultures, lower extremity, microbiology, sensitivity, specificity, wound.

While a general consensus exists that surface wound cultures have less utility than deeper cultures, surface cultures are nevertheless routinely used to guide empiric antibiotic administration. This is due in part to the ease with which surface cultures are obtained and the delay in obtaining deeper wound and bone cultures. The Infectious Diseases Society of America (IDSA) recommends routine culture of all diabetic infections before initiating empiric antibiotic therapy, despite caveats regarding undebrided wounds. ${ }^{1}$ Further examination of 2 additional societies, the European Society of Clinical Microbiology and Infectious Diseases and the Australasian Society for Infectious Diseases, reveals that they also do not describe guidelines on the role of surface wound cultures in skin, and skin structure infection (SSSI) management. ${ }^{2,3}$

Surface wound cultures are used to aid in diagnosis and appropriate antibiotic treatment of lower extremity foot ulcers. ${ }^{4}$ Contaminated cultures from other body locations have shown little utility and may be wasteful of resources. ${ }^{5,6}$ We hypothesize that given commensal skin flora, coupled with the additional flora that colonizes (chronic) lower ex- tremity wounds, surface wound cultures provide poor diagnostic accuracy for determining the etiology of acute infection. In contrast, many believe that deep tissue cultures obtained at time of debridement or surgical intervention may provide more relevant information to guide antibiotic therapy, thus serving as a gold standard. ${ }^{1-3,7,8}$ Nevertheless, with the ease of obtaining these superficial cultures and the promptness of the results, surface wound cultures are still used as a surrogate for the information derived from deeper cultures.

\section{Purpose}

The frequency at which superficial wound cultures correlate with the data obtained from deeper cultures is needed to interpret the posttest likelihood of infection. However, the sensitivity and specificity of superficial wound culture as a diagnostic test is unclear. The purpose of this study is to conduct a systematic review of the existing literature in order to investigate the relationship between superficial wound cultures and the etiology of SSSI. Accordingly, we 


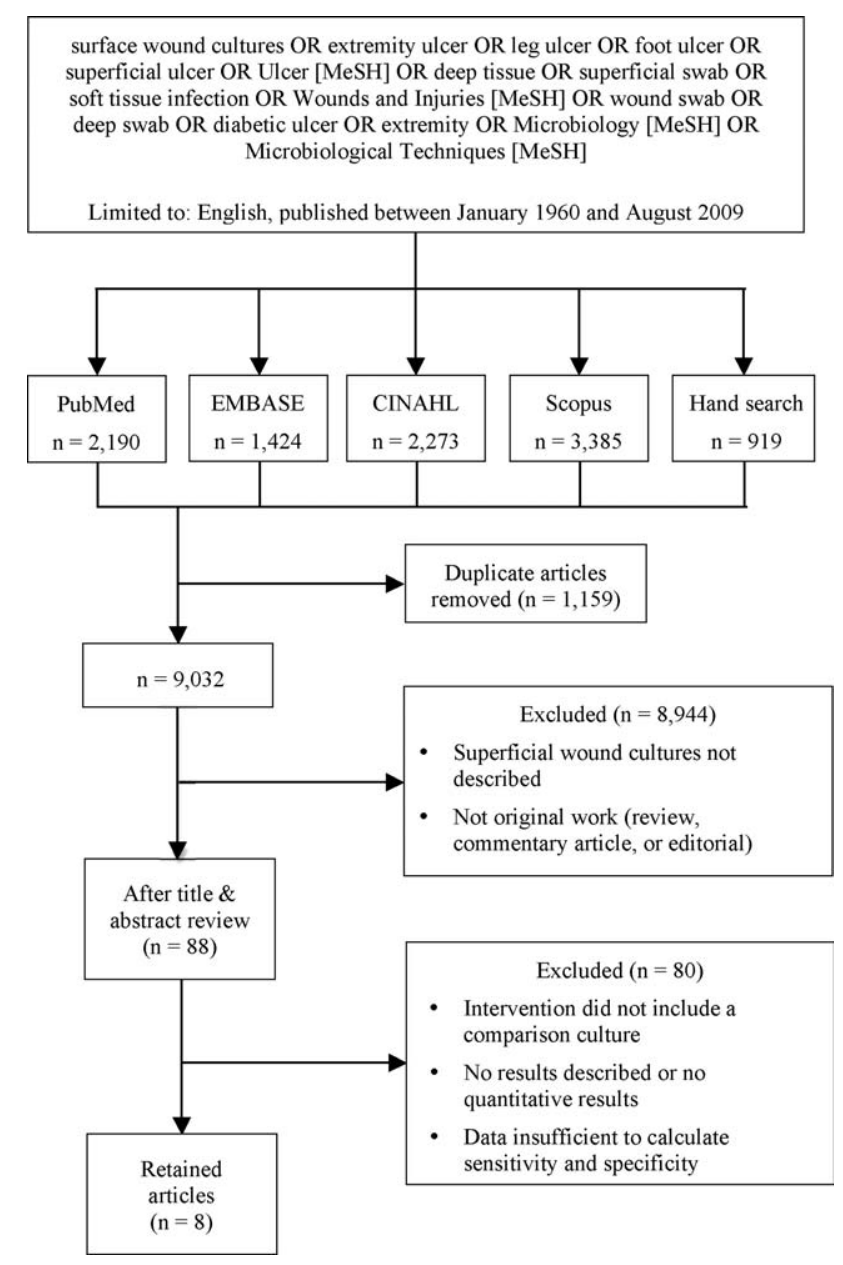

FIGURE 1. Flowchart of search strategy.

aim to describe any role that surface wound cultures may play in the treatment of lower extremity ulcers.

\section{Materials and Methods}

\section{Data Sources}

We identified eligible articles through an electronic search of the following databases: Medline through PubMed, Excerpta Medica Database (EMBASE), Cumulative Index of Nursing and Allied Health Literature (CINAHL), and Scopus. We also hand searched the reference lists of key review articles identified by the electronic search and the reference lists of all eligible articles (Figure 1).

\section{Study Selection}

The search strategy was limited to English articles published between January 1960 and August 2009. A PubMed search identified titles that contained the following keywords combined with $O R$ : surface wound cultures, extremity ulcer, leg ulcer, foot ulcer, superficial ulcer, Ulcer [MeSH], deep tissue, superficial swab, soft tissue infection, Wounds and Injuries $[\mathrm{MeSH}]$, wound swab, deep swab, diabetic ulcer, Microbiology [MeSH], Microbiological Techniques [MeSH]. Medical Subject Headings [MeSH] were used as indicated and were exploded to include subheadings and maximize results. This search strategy was adapted to search the other databases.

\section{Data Extraction}

Eligible studies were identified in 2 phases. In the first phase, 2 authors (AY and CC) independently reviewed potential titles of citations for eligibility. Citations were returned for adjudication if disagreement occurred. If agreement could not be reached, the article was retained for further review. In the second phase, 2 authors (AY and $\mathrm{CC}$ ) independently reviewed the abstracts of eligible titles. In situations of disagreement, abstracts were returned for adjudication and if necessary were retained for further review. Once all eligible articles were identified, 2 reviewers (AY and CL) independently abstracted the information within each article using a pre-defined abstraction tool. A third investigator (CC) reviewed all the abstracted articles for verification.

We initially selected articles that involved lower extremity wounds. Articles were included if they described superficial wound cultures along with an alternative method of culture for comparison. Alternative culture methods were defined as cultures derived from needle aspiration, wound base biopsy, deep tissue biopsy, surgical debridement, or bone biopsy. Further inclusion criteria required that articles have enough microbiology data to calculate sensitivity and specificity values for superficial wound swabs.

For the included articles, 2 reviewers (AY, CC) abstracted information pertaining to microbiology data from superficial wound swabs and alternative comparison cultures as reported in each article in the form of mean number of isolates recovered. Study characteristics and patient demographics were also recorded.

When not reported in the article, calculation of test sensitivity and specificity involved identifying true and falsepositive tests as well as true and false-negative tests. Articles were excluded if they did not contain sufficient data to calculate true/false-positive and true/false-negative tests. For all articles, we used the formulae [(sensitivity) $\div$ (1-specificity)] and [(1-sensitivity) $\div$ (specificity)] to calculate positive and negative likelihood ratios (LRs), respectively.

\section{Data Synthesis and Statistical Analysis}

Test sensitivity, specificity, positive and negative LR from all articles were pooled using a random-effects meta-analysis model (DerSimonian and Laird method). This method considers heterogeneity both within and between studies to calculate the range of possible true effects. ${ }^{9}$ For situations in which significant heterogeneity is anticipated, the randomeffects model is most conservative and appropriate. ${ }^{9,10}$

We also compared the mean number of organisms isolated from wound cultures to the mean number of organisms isolated from alternative culture methods using the nonparametric Wilcoxon rank sum test. Inter-rater reliability was assessed using the kappa statistic. We assessed potential 
TABLE 1. Sensitivities, Specificities, Positive and Negative Likelihood Ratios Calculated from Each Eligible Study

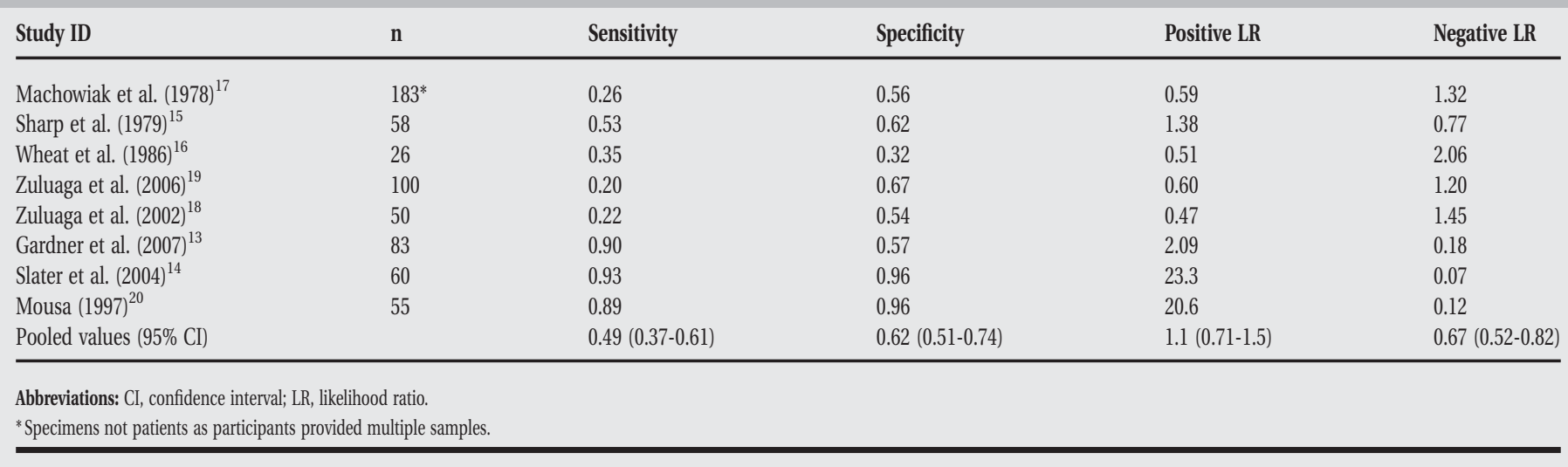

publication bias by visually examining a funnel plot as described by Egger et al. ${ }^{11}$ We report $95 \%$ confidence intervals, medians with interquartile ranges, and $p$-values where appropriate. All data analyses were performed using Stata 9.2 (STATA Corporation, College Station, TX, 2007).

\section{Results}

Of 9032 unique citations, eight studies met all inclusion criteria (Figure 1). Inter-rater reliability was substantial (Kappa $=0.78) .{ }^{12}$ Areas of initial disagreement generally involved whether a study adequately described an appropriate alternative culture method for comparison or whether data available in an article was sufficient for sensitivity and specificity calculation. Consensus was achieved once the full article was retrieved, reviewed and discussed.

The 8 studies evaluated in the review included a total number of 615 patients or samples (Table 1). Diabetic wounds were described in four studies. ${ }^{13-16}$ Two studies described wounds associated with peripheral vascular disease, ${ }^{13,17}$ while four involved traumatic wounds. ${ }^{13,17-19}$ One study did not identify the clinical circumstances concerning the wounds. ${ }^{20}$

The studies used several different methods for obtaining superficial cultures. Six studies obtained purulent wound drainage material through the application of sterile swabs. ${ }^{13-16,18,19}$ One study obtained purulent drainage material using needle aspiration. ${ }^{18}$ Two studies obtained culture material from sinus tracts associated with the wounds, one through sinus tract washings ${ }^{17}$ and another by obtaining sinus tract discharge material. ${ }^{20}$

The types of comparison cultures used were equally divided between deep tissue biopsies ${ }^{13-16}$ and bone biopsies, ${ }^{17-20}$ each accounting for $50 \%$ (4 of 8) of studies.

In assessing the data from the eight studies, the pooled test sensitivity for superficial wound swabs was $49 \%(95 \%$ confidence interval $[\mathrm{CI}], 37-61 \%$ ) (Figure 2). The pooled specificity for superficial wound swabs was $62 \%$ (95\% CI, $51-74 \%)$, while the pooled positive and negative LRs were 1.1 (95\% CI, 0.71-1.5) and 0.67 (95\% CI, 0.52-0.82), respectively (Figure 2).
The median number of bacterial isolates reported for each culture type, superficial and comparison culture, was collected from each study (Table 2). The median value for number of bacterial isolates identified by superficial culture was 2.7 (interquartile range [IQR] 1.8-3.2). The median value for number of bacterial isolates identified by comparison culture was 2.2 (IQR 1.7-2.9). A Wilcoxon rank sum analysis showed that the number of isolates for surface wound cultures was not significantly different than the number of isolates for comparison cultures $(P=0.75)$ (Table 1).

\section{Discussion}

In performing this review, we discovered ambiguity in the literature regarding the utility of surface wound cultures. Some studies obtained findings to suggest the utility of surface wound cultures, ${ }^{8,14,17}$ while other studies in our review $^{16,18,19}$ provided evidence against them. This variability confirmed the need for a meta-analytic approach as provided by this review.

While we have tried to minimize bias through a wellestablished methodology, we acknowledge that certain methodological limitations should be considered in interpreting the results. There may be publication bias in reviews that include only published articles; a funnel plot of sensitivity vs. sample size showed some asymmetry, suggesting bias. Our search strategy was limited to English-only articles, which may result in publication bias.

Further, this review included a group of studies that were heterogeneous in several regards. Differences exist in culturing methods and laboratory technology, as exemplified by the variety of superficial culture methods used. We were not able to account for these laboratory differences, as methodologies in obtaining and isolating bacteria were not uniformly well described.

Additionally, the studies classified organisms in different ways. Three studies categorized organisms according to Gram's stain characteristics. ${ }^{13,16,18}$ One study described organisms primarily in terms of aerobic or anaerobic respiration. ${ }^{15}$ Two studies ${ }^{14,19}$ discussed pathogens both in terms 


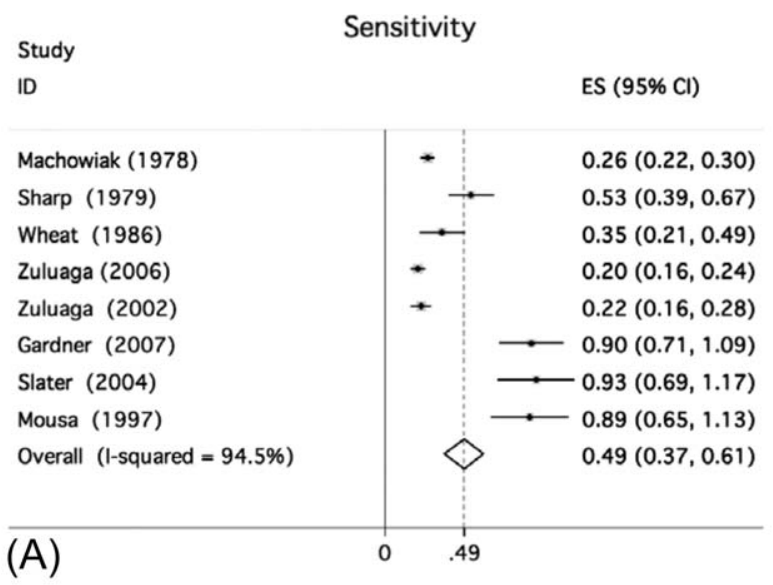

Study

ID
Specificity

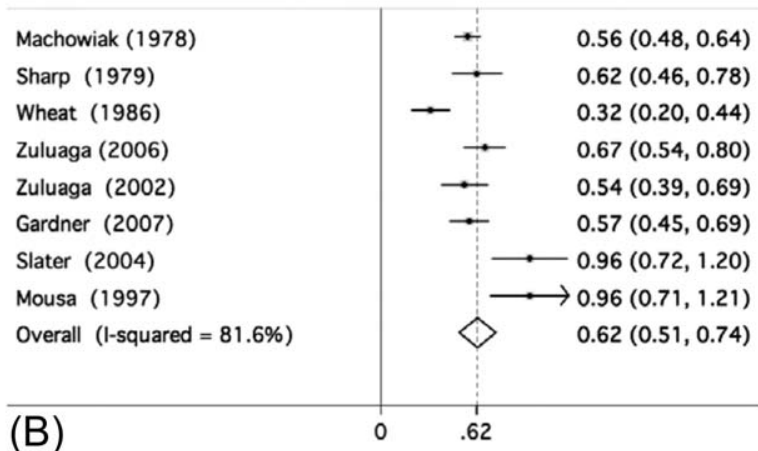

Negative Likelihood Ratio

\begin{tabular}{l|l} 
Study \\
ID \\
\hline Machowiak (1978) & $0.60(0.51,0.69)$ \\
Sharp (1979) & $1.40(1.05,1.75)$ \\
Wheat (1986) & $0.50(0.30,0.70)$ \\
Zuluaga (2006) & $0.60(0.48,0.72)$ \\
Zuluaga (2002) & $0.50(0.37,0.63)$ \\
Gardner (2007) & $2.10(1.65,2.55)$ \\
Slater (2004) & $23.30(17.42,29.18)$ \\
Mousa (1997) & $1.08(0.71,1.45)$ \\
Overall (1-squared = 96.0\%) & \\
\hline (C) & 01.1
\end{tabular}

\begin{tabular}{l|l} 
Study \\
ID
\end{tabular}

FIGURE 2. Forest plots created using a random-effects model for pooled sensitivity, (A) specificity, (B) positive likelihood ratio, (C) and negative likelihood ratio (D) regarding superficial wound cultures.

\begin{tabular}{|c|c|c|c|}
\hline Study ID & \# of Isolates (Swab) & \# of Isolates (Comparison) & Prior Antibiotics? \\
\hline Machowiak et al. $(1978)^{17}$ & $-^{*}$ & $-^{*}$ & Treated, but details not reported \\
\hline Sharp et al. $(1979)^{15}$ & 2.3 & 2.2 & Treated, but details not reported \\
\hline Wheat et al. $(1986)^{16}$ & 3.3 & 3.4 & Not described \\
\hline Zuluaga et al. (2006) ${ }^{19}$ & 1.3 & 1.6 & Antibiotics stopped 48 hours prior \\
\hline Zuluaga et al. (2002) ${ }^{18}$ & 1.1 & 1.4 & $52 \%$ on antibiotics, stopped 48 hours prior \\
\hline Gardner et al. $(2007)^{13}$ & 3.0 & 3.1 & $42 \%$ on antibiotics \\
\hline Slater et al. $(2004)^{14}$ & 2.7 & 2.5 & $27 \%$ on prior antibiotics \\
\hline Mousa $(1997)^{20}$ & 3.6 & 1.9 & Treated, but details not reported \\
\hline Median (IQR) & $2.7(1.8-3.2)$ & $2.2(1.7-2.9)$ & \\
\hline \multicolumn{4}{|c|}{ Abbreviation: IQR, interquartile range. } \\
\hline *Not reported within article. & & & \\
\hline
\end{tabular}

of respiration (aerobic/anaerobic) and Gram's stain characteristic, while another 2 studies $^{17,20}$ did not describe organisms in either terms. These inconsistencies limited our ability to provide sensitivity and specificity information for specific subclasses of organisms.

The clinical conditions in each study surrounding the wounds were also heterogeneous: most significantly in the issue of prior antibiotic administration. All but 1 study $^{16}$ indicated that the patients had received antibiotics prior to having cultures obtained. The type of antibiotics (narrowspectrum or broad-spectrum), the route of administration, and the cessation of antibiotics in relation to obtaining swabs and cultures all varied widely or were not well described. This degree of ambiguity will necessarily impact 
both the reliability of data regarding microbial growth as well as the component flora.

The inclusion of higher quality studies is likely to result in a more reliable meta-analysis. ${ }^{21}$ We had hoped that antibiotic trials would contain uniform outcomes and thus strengthen our meta-analysis through the inclusion of randomized-controlled studies. Unfortunately, the majority of antibiotic trials did not use superficial wound cultures, did not report mean number of isolates, or did not provide microbiological data in sufficient detail to calculate concordance rates-and therefore, did not meet eligibility criteria. Randomized-controlled trials were a minority among our included articles; the majority of study designs were retrospective cohorts and case-controlled studies.

Despite these limitations, we were able to conclude that superficial wound culture provides mediocre sensitivity $(49 \%)$ and specificity $(62 \%)$. The positive LR of 1.1 is unhelpful in decision making, having a CI that includes 1 . Interestingly, the negative LR of 0.67 could be somewhat helpful in medical decision making, modifying the pretest probability and assisting in ruling out a deeper bacterial infection. Although, according to Fagan's nomogram, a negative LR of 0.67 has only a mild effect on pretest odds. $^{22}$

The bacterial bioburden assessed by the number of isolates obtained by culture method serves as a proxy for reliability of culture results ${ }^{14,23}$ by suggesting that fewer organisms isolated from deep tissue or bone samples reflects a less contaminated specimen. Our assessment of the bioburden found that the median number of isolates was slightly higher in surface cultures than deeper cultures, though not to a significant degree $(P=0.75)$. This indicates that the degree of contamination in superficial cultures was neither significantly worse nor better than deep cultures.

We attempted to define a role for surface wound cultures; however, we found that these did not show any greater utility than deep cultures for identifying the microbiologic etiology of diabetic wound infections. While the negative LR provides some quantitative verification of the common clinical practice that a negative culture argues against infection, the finding is not especially robust.

Although for this meta-analysis we grouped all organisms in the same way, we recognize that the sensitivity and specificity may differ according to various subclasses of bacteria. Interpretations of culture results also vary (eg, Gram positive vs. negative; aerobic vs. anaerobic); practitioners will not interpret superficial cultures of coagulase-negative Staphylococcus in the same way as Pseudomonas. However, this study seeks to establish a reasonable starting point for the medical decision-making process by providing quantitative values in an area with previously conflicting data. We anticipate that as laboratory techniques improve and research into superficial wounds continues, greater sensitivity of superficial wound cultures will result.

Ultimately, physicians use culture data to target therapy in an effort to use the least toxic and most effective antimi- crobial agent possible to successfully treat infections. Clinical outcomes were not described in all included articles; in those that did, the endpoints were too dissimilar for meaningful comparison. Limiting our review to studies reporting treatment outcomes would have resulted in too few included studies. Thus, we were unable able to assess whether superficial wound cultures were associated with improved patient-oriented outcomes in this meta-analysis.

There is a significant paucity of trials evaluating the accurate concordance of superficial swabs to deep tissue culture. The current data shows poor sensitivity and specificity of superficial culture methods. The presumption that deeper cultures (such as a bone biopsy) should result in a less contaminated sample and more targeted culture results was also not borne out in our review. When presented with a patient with a wound infection, physicians mentally supply a pretest (or a pretreatment) probability as to the microbiologic etiology of the infection. Careful history will, of course, be critical in identifying extenuating circumstance or unusual exposures. From our meta-analysis, we cannot recommend the routine use of superficial wound cultures to guide initial antibiotic therapy as this may result in poor resource utilization. ${ }^{5}$ While clinical outcomes from the use of routine superficial cultures are unclear, we suggest greater use of local antibiograms and methicillin-resistant Staphylococcus aureus (MRSA) prevalence data to determine resistance patterns and guide the selection of empiric therapies.

\section{Address for correspondence and reprint requests:}

Chayan Chakraborti, MD, Section of General Internal Medicine and Geriatrics, Tulane University Health Sciences Center, 1430 Tulane Avenue, SL-16, New Orleans, LA 70112; Telephone: 504988-7518; Fax: 509-472-3758; E-mail:

cchakra@gmail.com Received 24 September 2009; revision received 14 January 2010; accepted 30 January 2010.

\section{References}

1. Lipsky BA, Berendt AR, Deery HG, et al. Diagnosis and treatment of diabetic foot infections. Clin Infect Dis. 2004;39:885-910.

2. AASID, Australasian Society for Infectious Diseases-Standards, Practice Guidelines (Skin and Soft Tissue Infections): Institute for Safe Medication Practices; 2006.

3. ESCMID, European Society of Clinical Microbiology \& Infectious Diseases-Standards, Practice Guidelines (Skin and Soft Tissue Infections): Institute for Safe Medication Practices; 2006.

4. Moran GJ, Amii RN, Abrahamian FM, Talan DA. Methicillin-resistant Staphylococcus aureus in community-acquired skin infections. Emerg Infect Dis. 2005;11:928-930.

5. Bates DW, Goldman L, Lee TH. Contaminant blood cultures and resource utilization. The true consequences of false-positive results. JAMA. 1991; 265:365-369.

6. Perl B, Gottehrer NP, Raveh D, Schlesinger Y, Rudensky B, Yinnon AM. Cost-effectiveness of blood cultures for adult patients with cellulitis. Clin Infect Dis. 1999;29:1483-1488.

7. Eron LJ, Lipsky BA, Low DE, Nathwani D, Tice AD, Volturo GA. Managing skin and soft tissue infections: expert panel recommendations on key decision points. J Antimicrob Chemother. 2003;52 Suppl 1:i3-i17. 
8. Pellizzer G, Strazzabosco M, Presi S, et al. Deep tissue biopsy vs. superficial swab culture monitoring in the microbiological assessment of limbthreatening diabetic foot infection. Diabet Med. 2001;18:822-827.

9. DerSimonian R, Laird N. Meta-analysis in clinical trials. Control Clin Trials. 1986;7:177-188.

10. Lau J, Ioannidis JP, Schmid CH. Quantitative synthesis in systematic reviews. Ann Intern Med. 1997;127:820-826.

11. Egger M, Davey Smith G, Schneider M, Minder C. Bias in meta-analysis detected by a simple, graphical test. BMJ. 1997;315:629-634.

12. Altman DG. Practical statistics for medical research. London, UK: Chapman \& Hall; 1991:403-409.

13. Gardner SE, Frantz RA, Saltzman CL, Hillis SL, Park H, Scherubel M. Diagnostic validity of three swab techniques for identifying chronic wound infection. Wound Repair Regen. 2006;14(5):548-57.

14. Slater RA, Lazarovitch T, Boldur I, et al. Swab cultures accurately identify bacterial pathogens in diabetic foot wounds not involving bone. Diabet Med. 2004;21:705-709.

15. Sharp CS, Bessmen AN, Wagner FW Jr, Garland D, Reece E. Microbiology of superficial and deep tissues in infected diabetic gangrene. Surg Gynecol Obstet. 1979;149:217-219.
16. Wheat LJ, Allen SD, Henry M, et al. Diabetic foot infections. Bacteriologic analysis. Arch Intern Med. 1986;146:1935-1940.

17. Mackowiak PA, Jones SR, Smith JW. Diagnostic value of sinus-tract cultures in chronic osteomyelitis. JAMA. 1978;239:2772-2775.

18. Zuluaga AF, Galvis W, Jaimes F, Vesga O. Lack of microbiological concordance between bone and non-bone specimens in chronic osteomyelitis: an observational study. BMC Infect Dis. 2002;2:8.

19. Zuluaga AF, Galvis W, Saldarriaga JG, Agudelo M, Salazar BE, Vesga O. Etiologic diagnosis of chronic osteomyelitis: a prospective study. Arch Intern Med. 2006;166:95-100.

20. Mousa HA. Evaluation of sinus-track cultures in chronic bone infection. J Bone Joint Surg Br. 1997;79:567-569.

21. Stroup DF, Berlin JA, Morton SC, et al. Meta-analysis of observational studies in epidemiology: a proposal for reporting. JAMA. 2000;283: 2008-2012.

22. Fagan TJ, Letter: nomogram for Bayes theorem. N Engl J Med. 1975;293: 257.

23. Bill TJ, Ratliff CR, Donovan AM, Knox LK, Morgan RF, Rodeheaver GT. Quantitative swab culture versus tissue biopsy: a comparison in chronic wounds. Ostomy Wound Manage. 2001;47:34-37. 